\title{
TABLES OF ABBREVIATIONS
}

\section{Texts}

A ................. Acts of the Apostles

Achilleid ........... L'Achilléide byzantine, ed . D. C. Hesseling (Amsterdam, 1919) ( $\mathrm{L}=$ The London Version, $N=$ The Naples Version)

C ................ Colossians (NT)

$1 \mathrm{C} \ldots \ldots \ldots \ldots \ldots \ldots \ldots \ldots \ldots \ldots \ldots \ldots$ Corinthians (NT)

2 C ............. 2 Corinthians (NT)

1 Chr. ............. 1 Chronicles (LXX)

2 Chr. ............ 2 Chronicles (LXX)

Chr. Mor. ........... The Chronicle of Morea, ed. J. Schmitt (London, 1904)

Da. ............... Daniel (LXX)

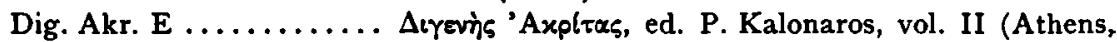
1941) (The Escorial Version)

Dig. Akr. GF ......... Digenes Akrites, ed. J. Mavrogordato (Oxford, 1956) (The Grottaferrata Version)

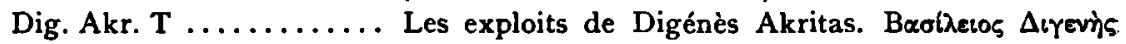
'Axplins, ed. C. Sathas and E. Legrand (Paris, 1875) (The Trebizond Version)

Dt. ............... Deuteronomy (LXX)

Dukas ............. Constantinus Ducas, ed. I. Bekker (Bonn, 1834)

E ............... Ephesians (NT)

Ex. ............... Exodus (LXX)

G .................. Galatians (NT)

Gen. ................ Genesis (LXX)

Georg. Cedr. .......... Georgius Cedrenus, ed. I. Bekker (Bonn, 1838-39)

Georg. M. ........... Georgius Monachus, ed. C. de Boor (Leipzig, 1892)

Georg. S. . ........... Georgius Syncellus, ed. G. Dindorf (Bonn, 1829)

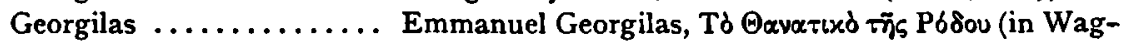
ner's Carmina, s. s. v.)

Glykas ............. Michael Glykas, ed. I. Bekker (Bonn, 1836)

H $\ldots \ldots \ldots \ldots \ldots \ldots$ Hebrews (NT)

Is. ............. Isaiah ( $\mathrm{LXX})$

Ja. ............... James (NT)

Jer. .............. Jeremiah (LXX)

Jn. $\ldots \ldots \ldots \ldots \ldots \ldots$ John $(\mathrm{NT})$

Jn. Sic. ............ Die Chronik des Johannes Sikeliota, ed. A. Heinrich (Graz, 1892)

Kekaum. ............ Cecaumeni Strategicon, ed. B. Wassiliewsky and V. Jernstedt (Petrograd, 1896) 
$1 \mathrm{Km} . \ldots \ldots \ldots \ldots \ldots \ldots$ Kingdoms (LXX)

$2 \mathrm{Km} . \ldots \ldots \ldots \ldots \ldots \ldots 2$ Kingdoms (LXX)

Leo Gramm............ Leo Grammaticus, ed. I. Bekker (Bonn, 1842)

Leont. N. . . . . . . . . Leontius of Naples, Vita Ioannis, ed. H. Gelzer (Freiburg-Leipzig, 1893)

Lev. ............. Leviticus ( $\mathrm{LXX}$ )

Libistros .......... Le Roman de Libistros et Rhodamné, ed. J. A. Lambert (Amsterdam, 1935)

Lk. .............. Luke (NT)

LXX ............. Septuagint

3 Macc. ........... 3 Maccabees (LXX)

Mal. ............. Ioannes Malalas, ed. L. Dindorf (Bonn, 1831)

Mk. ............. Mark (NT)

Mt. .............. Matthew (NT)

NT $\ldots \ldots \ldots \ldots \ldots \ldots$ New Testament

$1 \mathrm{P} \ldots \ldots \ldots \ldots \ldots \ldots \ldots \ldots \ldots \ldots$ Peter (NT)

$2 \mathrm{P} \ldots \ldots \ldots \ldots \ldots \ldots \ldots \ldots \ldots \ldots$ Peter (NT)

Pasch. Chr. .......... Chronicon Paschale, ed. L. Dindorf (Bonn, 1832)

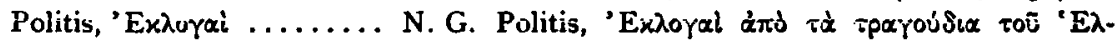

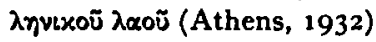

Porph. Adm. .......... Constantine Porphyrogenitus, De Administrando Imperio, ed. Gy. Moravcsik-R. Jenkins (Budapest, 1949)

Porph. Cer. .......... Constantinus Porphyrogenitus, De Ceremoniis Aulae Byzantinae, ed. I. Reiske (Bonn, 1829-30)

Procop. ............ Procopius, De Aedificils, ed. J.Haury (Leipzig, 1913)

Prodr. poems .......... Poèmes Prodromiques en grec vulgaire, ed. D. C. Hesseling and H. Pernot (Amsterdam, 1910)

Ps. .............. Psalms (LXX)

R $\ldots \ldots \ldots \ldots \ldots \ldots \ldots$ Romans (NT)

Rev. ............. Revelation (NT)

Sir. .............. Jesus Sirach $(\mathrm{LXX})$

$\mathrm{T} \ldots \ldots \ldots \ldots \ldots \ldots \ldots$ Titus (NT)

$1 \mathrm{~T} \ldots \ldots \ldots \ldots \ldots \ldots \ldots$ Timothy $(\mathrm{NT})$

1 Th. $\ldots \ldots \ldots \ldots \ldots \ldots 1$ Thessalonians (NT)

2 Th. $\quad \ldots \ldots \ldots \ldots \ldots \ldots 2$ Thessalonians (NT)

Theod. ............ Theodosius Melitenus, ed. G. Tafel (Munich, 1859)

Theoph. ............ Theophanes Confessor, ed. C. de Boor (Leipzig, 1883-85)

Theoph. cont. ......... Theophanes continuatus, ed. I. Bekker (Bonn, 1838)

Wagner, Carmina ....... G. Wagner, Carmina Graeca Medii Aevi (Leipzig, 1874)

\section{Literature}

BAG

A Greek-English Lexicon of the New Testament and Other Early Christian Literature [A translation and adaptation of W. Bauer's "Griechisch-Deutsches 
Wörterbuch zu den Schriften des Neuen Testaments und der übrigen urchristlichen Literatur" by W. F. Arndt and F. W. Gingrich (Cambridge and Chicago, 1957)]

Bănescu ............ N. Bănescu, Die Entwicklung des griechischen Futurums von der frühbyzantinischen Zeit bis zur Gegenwart (Bucharest, 1915)

BDF ............. F. Blass and A. Debrunner, A Greek Grammar of the New Testament and Other Early Christian Literature [A translation and revision of F. Blass and A. Debrunner's "Grammatik des neutestamentlichen Griechisch" incorporating supplementary notes of A. Debrunner by R. W. Funk (Cambridge and Chicago, 1961)]

Böhlig ........... G. Böhlig, Untersuchungen zum rhetorischen Sprachgebrauch der Byzantiner mit besonderer Berücksichtigung der Schriften des Michael Psellos (Berlin, 1956)

Campbell ........... J. M. Campbell, The Influence of the Second Sophistic on the Style of the Sermons of St. Basil the Great (Washington D. C., 1922) (The Catholic University of America, Patristic Studies, II)

Christensen ........... H. Christensen, Die Sprache des byzantinischen Alexandergedichtes (Byzantinische Zeitschrift 7, $1898,366 \mathrm{f}$.)

de Matons ........... J. Grosdidier de Matons, Romanos le Mélode. Hymnes, I-IV (Paris, 1964-67) (Sources Chrétiennes, 99, $110,114,128)$

Debrunner ........... A. Debrunner, Geschichte der griechischen Sprache, II. Grundfragen und Grundzüge des nachklassischen Griechisch (Berlin, 1954) (Sammlung Göschen, 114)

Deißmann ........... A. Deißmann, Bibelstudien (Marburg, 1895)

Deißmann, N B . . . . . . . A. Deißmann, Neue Bibelstudien (Marburg, 1897)

Denniston .......... J. D. Denniston, Greek Prose Style (Oxford, 1952)

Dieterich ............. Kieterich, Untersuchungen zur Geschichte der griechischen Sprache von der hellenistischen Zeit bis zum 10. Jahrhundert n. Chr. (Leipzig, 1898) (Byzantinisches Archiv, 1)

Du Cange ........... Carolus du Fresne Du Cange, Glossarium ad Scriptores mediae et infimae Graecitatis (Lyon, 1688)

Hatzidakis, Einleitung ... G. Hatzidakis, Einleitung in die neugriechische Grammatik (Leipzig, 1892)

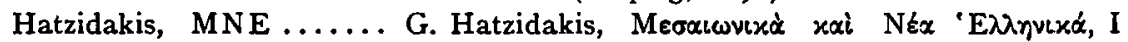
(Athens, 1905), II (1907)

Helbing ........... R. Helbing, Die Kasussyntax der Verba bei den Septuaginta. Ein Beitrag zur Hebraismenfrage und zur Syntax der Koเvý (Göttingen, 1928) 
Helbing, Grammatik .... R. Helbing, Grammatik der Septuaginta. Laut- und Wortlehre (Göttingen, 1907)

Hesseling ............. s. Achilleid

Jannaris ............ A. N. Jannaris, An Historical Greek Grammar (London, 1897)

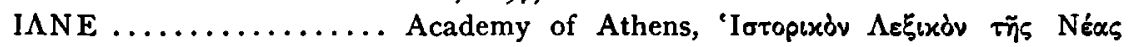

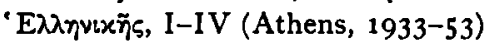

Kapsomenos, Voruntersuchungen .......... S. G. Kapsomenakis, Voruntersuchungen zu einer Grammatik der Papyri der nachchristlichen Zeit (Munich, 1938) (Münchener Beiträge zur Papyrusforschung und antiken Rechtsgeschichte, 28)

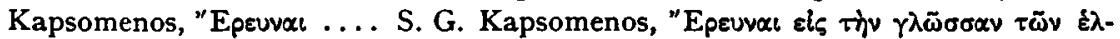

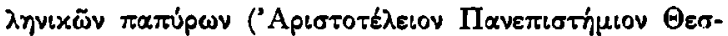

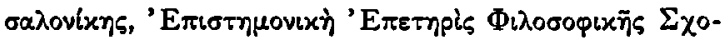
$\lambda \tilde{n} 57,1957,325-72$ )

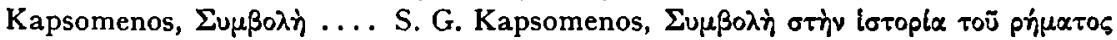

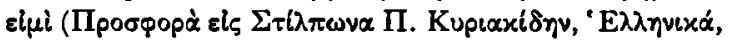
Supplement 4, Thessalonica, 1953, 305-25)

Kretschmer ......... P. Kretschmer, Die Entstehung der Koเvín (Sitzungsberichte der Wiener Akademie 143, 10 [1901])

Krumbacher, Hl. Theodosios .............. Krumbacher, Studien zu den Legenden des hl. Theodosios (Sitzungsberichte der philos.-philol. und histor. Klasse der K. Bayer. Akademie der Wissenschaften zu München, 1892, $272 \mathrm{f}$.)

Krumbacher, Studien .... K. Krumbacher, Studien zu Romanos (Sitzungsberichte der philos.-philol. und histor. Klasse der $\mathrm{K}$. Bayer. Akademie der Wissenschaften zu München, 1898, II, 69 f.)

Krumbacher, Umarbeitungen ............ Krumbacher, Umarbeitungen bei Romanos (Sitzungsberichte der philos.-philol. und histor. Klasse der K. Bayer. Akademie der Wissenschaften zu München, 1899, II, 1 f.)

Krumbacher, Romanos und Kyriakos ........ K. Krumbacher, Romanos und Kyriakos. Das Verhältnis des Liedes "Lazarus" von Kyriakos zum Liede "Judas" von Romanos (Sitzungsberichte der philos.-philol. und histor. Klasse der K. Bayer. Akademie der Wissenschaften zu München, 1901, 693 f.)

Krumbacher, Akrostichis . K. Krumbacher, Die Akrostichis in der griechischen Kirchenpoesie (Sitzungsberichte der philos.-philol. und histor. Klasse der K. Bayer. Akademie der Wissenschaften zu München, 1903, 551 f.)

Krumbacher, Miscellen ... K. Krumbacher, Miscellen zu Romanos (Abhandlungen der philos.-philol. Klasse der K. Bayer. Aka-

II Byzant. Archiv, Mitsakis 
demie der Wissenschaften zu München, 24, 1909, III, $1 \mathrm{f.})$

Krumbacher, Hl. Georg .. K. Krumbacher, Der hl. Georg in der griechischen Uberlieferung (Abhandlungen der philos.-philol. Klasse der K. Bayer. Akademie der Wissenschaften zu München, 25, 1911, III, $1 \mathrm{f}$.)

Kühner-Blass ......... R. Kühner, Ausführliche Grammatik der griechischen Sprache, 1. Elementar- und Formenlehre, 3. Auflage von $F$. Blass (Hannover, 1890-92) (in two vols.)

Kühner-Gerth ........ R. Kühner, Ausführliche Grammatik der griechischen Sprache, II. Satzlehre, 3. Auflage von B. Gerth (Hannover-Leipzig, 1898-1904; reprinted 1955)

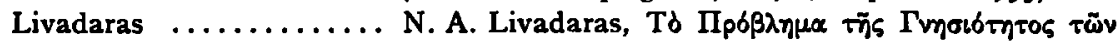

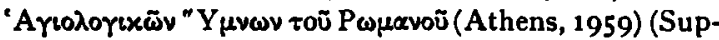

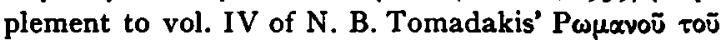

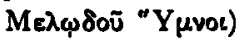

Lambert ............... s. Libistros

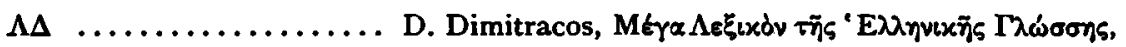
I-IX (Athens, 1933-51)

LSJ .............. A Greek-English Lexicon compiled by H. G. Liddell and $R$. Scott. A new edition revised by $H$. S. Jones (Oxford, 1925-40)

Maas, Chronologie ...... P. Maas, Die Chronologie der Hymnen des Romanos (Byzantinische Zeitschrift 15, 1906, 1 f.)

Maas, Umarbeitungen ... P. Maas, Grammatische und metrische Umarbeitungen in der Uberlieferung des Romanos (Byzantinische Zeitschrift 16, 1907, 565 f.)

Maas, Akklamationen .... P. Maas, Metrische Akklamationen der Byzantiner (Byzantinische Zeitschrift 21, 1912, $28 \mathrm{f}$.)

Maas, Weihnachtslied ... P. Maas, Das Weihnachtslied des Romanos (Byzantinische Zeitschrift 24, 1923-24, 1 f.)

Maas, Kirchenpoesie ..... P. Maas, Frühbyzantinische Kirchenpoesie. Anonyme Hymnen des V.-VI. Jahrhunderts (Berlin, $1931^{2}$ ) (Kleine Texte für Vorlesungen und Übungen, herausg. von $\mathrm{H}$. Lietzmann)

Maas-Trypanis, SRMC. .. P. Maas and C. A. Trypanis, Sancti Romani Melodi Cantica (Oxford, 1963)

Mayser ............. E. Mayser, Grammatik der griechischen Papyri aus der Ptolemäerzeit, I (Leipzig, 1906), II ${ }^{1}$ (Berlin-Leipzig, 1926), II ${ }^{2}$ (1933), II ${ }^{3}$ (1934); second edition: $I^{2}$ (1938), I3 (1936)

Mitsakis ............ K. Mitsakis, The Vocabulary of Romanos the Melodist (Glotta 43, 1965, $171 \mathrm{f}$ )

MM $\ldots \ldots \ldots \ldots \ldots$ The Vocabulary of the Greek Testament illustrated from the papyri and other nonliterary sources by J. $\mathrm{H}$. Moulton and G. Milligan (London, 1914-29) 
Moulton ........... J.H. Moulton, A Grammar of New Testament Greek, I. Prolegomena (Edinburgh, 1908), II. Accidence and Word-Formation with an Appendix on Semitisms in the New Testament (1919-29) (the second vol. with the collaboration of W. F. Howard)

Norden ............. E. Norden, Die Antike Kunstprosa (Stuttgart, 1958) Palmer ............ L. R. Palmer, A Grammar of the Post-Ptolemaic Papyri, I. Accidence and Word-Formation, Part I, The Suffixes (London, 1946) (Publications of the Philological Society)

Papadopoulos, 'E $\pi$ ip-

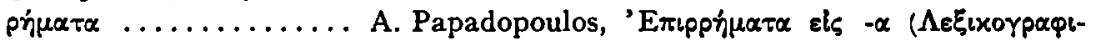

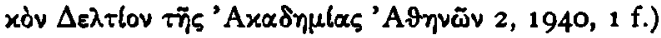

Papadopoulos, $\Lambda \varepsilon \xi \varepsilon\llcorner\varsigma$

'Aগno.

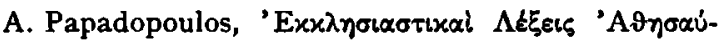

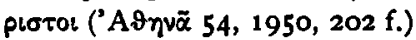

Pitra ............. J. B. Pitra, Analecta Sacra Spicilegio Solesmensi parata, I (Paris, 1876)

Psaltis ............ S. Psaltis, Grammatik der Byzantinischen Chroniken (Göttingen, 1913) (Forschungen zur griechischen und lateinischen Grammatik, 2)

Psaltis, 'Y

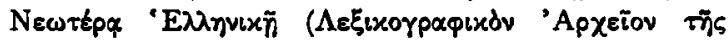

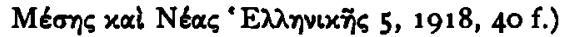

Radermacher ......... L. Radermacher, Koine (Sitzungsberichte der Wiener Akademie 224, 5 [1947])

Reinhold ............ H. Reinhold, De graecitate Patrum Apostolicorum librorumque apocryphorum Novi Testamenti quaestiones grammaticae (Dissertationes philologicae Hallenses XIV 1 [1898])

Sophocles..$\ldots \ldots \ldots \ldots$ E. A. Sophocles, Greek Lexicon of the Roman and Byzantine Periods from B.C. 146 to A. D. 1100 (Boston, 1870)

Tabachovitz, Studien .... D. Tabachovitz, Sprachliche und textkritische Studien zur Chronik des Theophanes Confessor (Uppsala, 1926)

Tabachovitz, Études .... D. Tabachovitz, Études sur le grec de la basse époque (Uppsala-Leipzig, 1943)

Thumb ............. A. Thumb, Handbuch der neugriechischen Volkssprache (Straßburg, 1910)

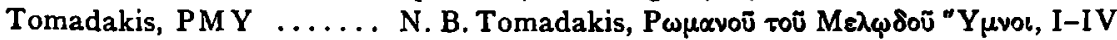
(Athens, 1952-61)

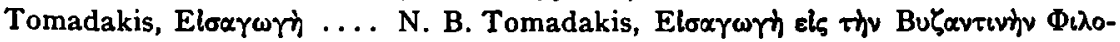

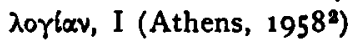

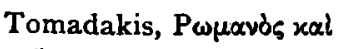

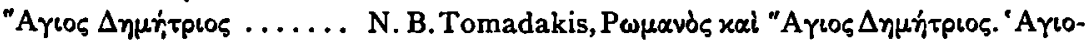

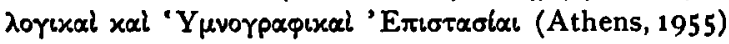


Triandaphyllidis

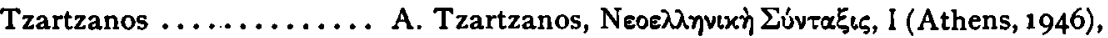
II (1953)

Wolf 1911), II (1912)

\title{
III. General and Special Abbreviations
}

\author{
acc. = accusative \\ act. $=$ active \\ A. D. = Anno Domini \\ adj. = adjective(s) \\ adv. $=$ adverb(s) \\ al. = alibi \\ aor. = aorist \\ B. C. $=$ Before Christ \\ B. Z. = Byzantinische Zeitschrift \\ Byz. = Byzantine \\ cf. $=$ confer \\ class. $=$ classical \\ $\operatorname{cod}(\mathrm{d}) .=\operatorname{codex}($-ices $)$ \\ constr. = constructed, construction \\ dat. $=$ dative \\ e. $g$. = exempli gratia \\ ed. = edited, edition \\ f. = following \\ fem. $=$ feminine \\ fn. = footnote \\ gen. = genitive \\ Hell. = Hellenistic \\ ibid. = ibidem \\ i. e. $=$ id est \\ indecl. = indeclinable \\ inf. = infinitive \\ intrans. $=$ intransitive
}

introd. $=$ introduction

K. Z. = Kuhn's Zeitschrift

l. = line

m. c. = metri causa

masc. $=$ masculine

M. Gr. = Modern Greek

mid. = middle

$\mathrm{ms}(\mathrm{s})=$ manuscript $(\mathrm{s})^{*}$

neut. $=$ neuter

$\mathrm{no}(\mathrm{s}) .=$ number $(\mathrm{s})$

nom. = nominative

$\mathrm{p}(\mathrm{p})$. = page(s)

pass. $=$ passive

pers. $=$ person

pl. = plural

ptcp. = participle

s. $\Rightarrow$ see

sg. = singular

s. v. = sub voce

subj. = subjunctive

trans. $=$ transitive

$\operatorname{vb}(\mathbf{s}) .=\operatorname{verb}(\mathbf{s})$

voc. $=$ vocative

$\operatorname{vol}(s) .=\operatorname{volume}(s)$

v. l. = varia lectio

$\mathrm{v}(\mathrm{s}),=\operatorname{verse}(\mathbf{s})$

* The following sigla are employed to indicate the mss of Romanos mentioned in the present treatise: $\mathrm{A}=$ Athous Batopediou 1041, saec. $\mathrm{X}-\mathrm{XI} ; \mathrm{B}=$ Athous Laurae $\Gamma$ 27, saec. $X-X I ; D=$ Athous Laurae $\Gamma$ 28, saec. $X I ; G=$ Sinaiticus 925 , saec. $X ; M=$ Mosquensis Synod. 437, saec. XII; $P=$ Patmiaci 212 and 213 , saec. XI; $T=$ Taurinensis $B$. iv. 34 , saec. $X I ; \Delta=(C=$ Corsinianus 366 , saec. $\mathrm{X}-\mathrm{XI}$ and $\mathrm{V}=$ Vindobonensis, suppl. gr. 96, saec. XII); $\mathrm{b}=$ Cryptoferratensis $\Delta$ a I, saec. XI-XII; s. Maas-Trypanis, SRMC, p. XXXV. 